\title{
On the migration of protoplanets embedded in circumbinary disks
}

\author{
A. Pierens and R. P. Nelson
}

\author{
Astronomy Unit, Queen Mary, University of London, Mile End Rd, London, E1 4NS, UK \\ e-mail: a.pierens@qmul.ac.uk
}

Received 17 April 2007 / Accepted 13 July 2007

\section{ABSTRACT}

\begin{abstract}
Aims. We present the results of hydrodynamical simulations of low mass protoplanets embedded in circumbinary accretion disks. The aim is to examine the migration and long term orbital evolution of the protoplanets, in order to establish the stability properties of planets that form in circumbinary disks.

Methods. Simulations were performed using a grid-based hydrodynamics code. First we present a set of calculations that study how a binary interacts with a circumbinary disk. We evolve the system for $\sim 10^{5}$ binary orbits, which is the time needed for the system to reach a quasi-equilibrium state. From this time onward the apsidal lines of the disk and the binary are aligned, and the binary eccentricity remains essentially unchanged with a value of $e_{\mathrm{b}} \sim 0.08$.

Once this stationary state is obtained, we embed a low mass protoplanet in the disk and let it evolve under the action of the binary and disk forces. We consider protoplanets with masses of $m_{\mathrm{p}}=5,10$ and $20 M_{\oplus}$.

Results. In each case, we find that inward migration of the protoplanet is stopped at the edge of the tidally truncated cavity formed by the binary. This effect is due to positive corotation torques, which can counterbalance the net negative Lindblad torques in disk regions where the surface density profile has a sufficiently large positive gradient. Halting of migration occurs in a region of long-term stability, suggesting that low mass circumbinary planets may be common, and that gas giant circumbinary planets should be able to form in circumbinary disks.
\end{abstract}

Key words. accretion, accretion disks - planets and satellites: formation - stars: binaries: close - hydrodynamics methods: numerical

\section{Introduction}

Among the 215 extrasolar planets discovered to date, about 30 of them reside in binary or multiple star systems (Eggenberger et al. 2004; Mugrauer et al. 2006; Boss 2006). Most of them are members of stellar binaries and orbit around one star, on so-called S-type orbits. In comparison with planets around single stars, the short-period planets in binary systems appear to be more massive (Zucker \& Mazeh 2002) and tend to a have a very low eccentricity when their period is shorter than about 40 days (Eggenberger et al. 2004).

Most of the binary systems harbouring planets have a binary separation $a_{\mathrm{b}} \geq 100 \mathrm{AU}$. However, planets orbiting at 1-2 AU from one star have also been detected in Gliese 86, $\gamma$ Cephei and HD 41004 A, which are binary systems with $a_{\mathrm{b}} \sim 20$ AU (Eggenberger et al. 2004; Mugrauer \& Neuhauser 2005). For shorter binary separations, a planet cannot orbit stably around one star because of the strong perturbations due to the binary companion. The largest stable planetary orbit $a_{\max }$ depends on the binary mass ratio $q_{\mathrm{b}}$ and eccentricity $e_{\mathrm{b}}$ (Holman \& Wiegert 1999). For a binary with mass ratio $q_{\mathrm{b}}=0.3$, we have $a_{\max } \sim 0.37 a_{\mathrm{b}}$ for $e_{\mathrm{b}}=0$ and $a_{\max } \sim 0.14 a_{\mathrm{b}}$ for $e_{\mathrm{b}}=0.5$ (Eggenberger et al. 2004).

In principle, close binaries with $a_{\mathrm{b}} \sim 1 \mathrm{AU}$ can harbour planets evolving on a stable P-type orbit which encircles the two components of the binary. The stability of such planets was studied by Holman \& Wiegert (1999), who provide empirically derived formulae for planetary stability depending on binary mass ratio and eccentricity. A circumbinary planet with mass $m_{\mathrm{p}}=2.5 M_{\mathrm{J}}$ has been detected orbiting at $23 \mathrm{AU}$ from the radio pulsar binary PSR 1620-26. Another with mass $m_{\mathrm{p}}=2.44 M_{\mathrm{J}}$ has been found to orbit around the system composed of the star HD 202206 and its 17.4 $M_{\mathrm{J}}$ companion (Udry et al. 2002). Because close binaries are often rejected from Doppler radial velocity surveys, planets have not yet been observed evolving on P-type orbits around main sequence binary stars.

However, several circumbinary disks have been observed around spectroscopic binaries like DQ Tau, AK Sco and GW Ori. In a few cases like GG Tau (Dutrey et al. 1994), the circumbinary disk has been directly imaged. Observations reveal that this disk is truncated at its inner edge, which is a consequence of the tidal torques exerted by the binary. The truncation radius is $\sim 2.7 a_{\mathrm{b}}$ which is consistent with analytical estimates from Artymowicz \& Lubow (1994). From analytical calculations and numerical simulations, we expect the truncation radius to range between $1.8 a_{\mathrm{b}}$ and $2.6 a_{\mathrm{b}}$, depending on the binary eccentricity (Artymowicz \& Lubow 1994; Günther \& Kley 2002; Günther et al. 2004).

Assuming that planets can form inside circumbinary disks, the fact that such disks have been observed and that $\sim 50 \%$ of the solar-type stars are members of binary or multiple star systems (Duquennoy \& Mayor 1991) suggests that circumbinary planets may be common. It is thus of interest to investigate how planet formation occurs in circumbinary disks. So far, very few studies have focused on this topic. Moriwaki \& Nakagawa (2004) studied planetesimal accretion in gas-free circumbinary disks, and found that planetesimals can grow only in regions farther out than 13 AU from a binary with $a_{\mathrm{b}}=1 \mathrm{AU}$ and $e_{\mathrm{b}}=0.1$. The influence of gas may be important here as it will provide eccentricity damping for the planetesimals, such that the region in which accretion can occur lies closer to the binary. Recently, 
Quintana \& Lissauer (2006) simulated the late stages of terrestrial planet formation for $0.05 \mathrm{AU} \leq a_{\mathrm{b}} \leq 0.4 \mathrm{AU}$ and $0<e_{\mathrm{b}}<0.8$. They found that planetary systems with properties similar to those around single stars can be formed around binaries with $a_{\mathrm{b}}\left(1+e_{\mathrm{b}}\right) \leq 0.2$ AU. Larger values of $a_{\mathrm{b}}$ and $/$ or $e_{\mathrm{b}}$ lead to sparser planetary systems.

Nelson (2003) studied the orbital evolution of a giant planet embedded in a circumbinary disk. This work indicated that giant planets could become trapped in mean motion resonances with the central binary. This was caused by the planet migrating through the disk due to type II migration. Subsequent evolution depended on whether the resonant trapping was stable. Unstable systems suffered the fate of being ejected by the binary, becoming free-floating planets while stable systems remained near or at the resonance.

Here, we are interested in an earlier stage of evolution during which the giant planet is still forming through the growth of a solid core. To address this issue, we have performed hydrodynamical simulations of low mass protoplanets evolving in a circumbinary disk. We begin by simulating the evolution of a binary-circumbinary disk system. These simulations are run for $\sim 10^{5}$ binary orbits until we get a quasi-stationary state, within which the disk structure and the binary eccentricity remain unchanged. We then use this equilibrium state as the initial conditions for the disk and the binary in subsequent simulations of protoplanets embedded in circumbinary disks.

This paper is organized as follows. In Sect. 2 we describe the physical model and the numerical setup. In Sect. 3 we present the results of the simulations. We first describe the results dealing with binary-disk interactions, and discuss these results in the context of previous theoretical and numerical work on binary-disk interactions. We then focus on the evolution of planets in circumbinary disks. We find that a low-mass protoplanet is trapped at the edge of the cavity created by the binary and we show that this effect arises when the corotation and Lindblad torques cancel each other. We finally discuss our results in Sect. 4 and present our conclusions.

\section{Equations of motion}

\subsection{Disk evolution}

Assuming that the disk aspect ratio is small, we can consider vertically averaged quantities when writing the equations of motion. The problem is therefore reduced to a two-dimensional one. In polar coordinates $(r, \phi)$ and in a frame with the origin located at the centre of mass of the binary, the continuity equation reads:

$\frac{\partial \Sigma}{\partial t}+\nabla \cdot(\Sigma \boldsymbol{v})=0$

where $\Sigma=\int_{-\infty}^{\infty} \rho \mathrm{d} z$ is the disk surface density. The equations for the radial and azimuthal components of the disk velocity $\boldsymbol{v}=$ $\left(v_{r}, v_{\phi}\right)$ are respectively given by:

$\frac{\partial \Sigma v_{r}}{\partial t}+\nabla \cdot\left(\Sigma v_{r} \boldsymbol{v}\right)-\frac{\Sigma v_{\phi}^{2}}{r}=-\frac{\partial p}{\partial r}-\Sigma \frac{\partial \Phi}{\partial r}+f_{r}$

and

$\frac{\partial \Sigma v_{\phi}}{\partial t}+\nabla \cdot\left(\Sigma v_{\phi} \boldsymbol{v}\right)+\frac{\Sigma v_{r} v_{\phi}}{r}=-\frac{1}{r} \frac{\partial p}{\partial \phi}-\frac{\Sigma}{r} \frac{\partial \Phi}{\partial \phi}+f_{\phi}$.

In the above equations, $p$ is the vertically integrated pressure, $f_{r}$ and $f_{\phi}$ are respectively the radial and azimuthal components of the vertically averaged viscous force per unit volume. Expressions for $f_{r}$ and $f_{\phi}$ can be found for example in
Nelson et al. (2000). $\Phi$ is the gravitational potential and can be written as:

$\Phi=\sum_{i=1}^{2} \Phi_{\mathrm{s} i}+\Phi_{\mathrm{p}}+\Phi_{\text {ind }}$

where $\Phi_{\mathrm{s} i}$ is the potential of the $i$ th member of the binary with mass $M_{\mathrm{si}}$ :

$\Phi_{\mathrm{s} i}=-\frac{G M_{\mathrm{s} i}}{\left|\boldsymbol{r}-\boldsymbol{r}_{\mathrm{s} i}\right|^{3}}$,

and $\Phi_{\mathrm{p}}$ is the potential of the planet with mass $m_{\mathrm{p}}$ :

$\Phi_{\mathrm{p}}=-\frac{G m_{\mathrm{p}}}{\sqrt{r^{2}+r_{\mathrm{p}}^{2}-2 r r_{\mathrm{p}} \cos \left(\phi-\phi_{\mathrm{p}}\right)+\epsilon^{2}}}$

In the previous equation, $\epsilon$ is a softening parameter and is chosen to be $\epsilon=0.6 H / r$, where $H / r$ is the disk aspect ratio. $\Phi_{\text {ind }}$ is an indirect term which comes from the fact that the frame centered on the binary centre of mass is not inertial. This term reads:

$\Phi_{\text {ind }}=G \sum_{i=1}^{2} \frac{M_{\mathrm{s} i}}{M_{*}}\left(\frac{m_{\mathrm{p}}\left(\boldsymbol{r}_{\mathrm{p}}-\boldsymbol{r}_{\mathrm{s} i}\right)}{\left|\boldsymbol{r}_{\mathrm{p}}-\boldsymbol{r}_{\mathrm{s} i}\right|^{3}}+\int_{\mathrm{S}} \frac{\mathrm{d} m\left(\mathbf{r}^{\prime}\right)\left(\boldsymbol{r}^{\prime}-\boldsymbol{r}_{\mathrm{s} i}\right)}{\left|\boldsymbol{r}^{\prime}-\boldsymbol{r}_{\mathrm{s} i}\right|^{3}}\right) \cdot \boldsymbol{r}$,

where $M_{*}$ is the total mass of the binary and the integral is performed over the surface area of the disk.

\subsection{Binary and planet orbital evolution}

In this work each body can experience the gravitational force due to every other one. In other words, we allow the planet to gravitationally interact with both the disk and the binary, while each member of the binary can interact with the other star and also with the disk and the planet. The equation of motion for the protoplanet is therefore given by:

$\frac{\mathrm{d}^{2} \boldsymbol{r}_{\mathrm{p}}}{\mathrm{d} t^{2}}=-\sum_{i=1}^{2} \frac{G M_{\mathrm{s} i}\left(\boldsymbol{r}_{\mathrm{p}}-\boldsymbol{r}_{\mathrm{s} i}\right)}{\left|\boldsymbol{r}_{\mathrm{p}}-\boldsymbol{r}_{\mathrm{si}}\right|^{3}}+\boldsymbol{f}_{\mathrm{dp}}-\nabla \Phi_{\text {ind }}$,

and the equation of motion for the $i$ th member of the binary is:

$\frac{\mathrm{d}^{2} \boldsymbol{r}_{\mathrm{s} i}}{\mathrm{~d} t^{2}}=-\frac{G M_{\mathrm{s} j}\left(\boldsymbol{r}_{\mathrm{s} i}-\boldsymbol{r}_{\mathrm{s} j}\right)}{\left|\boldsymbol{r}_{\mathrm{s} i}-\boldsymbol{r}_{\mathrm{s} j}\right|^{3}}-\frac{G m_{\mathrm{p}}\left(\boldsymbol{r}_{\mathrm{s} i}-\boldsymbol{r}_{\mathrm{p}}\right)}{\left|\boldsymbol{r}_{\mathrm{p}}-\boldsymbol{r}_{\mathrm{s} i}\right|^{3}}+\boldsymbol{f}_{\mathrm{d} i}-\nabla \Phi_{\text {ind }}$.

In the previous equations, $f_{\mathrm{d} i}$ is the force due to the disk and is defined by:

$\boldsymbol{f}_{\mathrm{d} i}=-G \int_{S} \frac{\Sigma\left(\boldsymbol{r}^{\prime}\right)\left(\boldsymbol{r}_{\mathrm{s} i}-\boldsymbol{r}^{\prime}\right) \mathrm{d} \boldsymbol{r}^{\prime}}{\left(r^{\prime 2}+r_{\mathrm{s} i}^{2}-2 r^{\prime} r_{\mathrm{s} i} \cos \left(\phi^{\prime}-\phi_{\mathrm{s} i}\right)+\epsilon^{2}\right)^{3 / 2}}$

Note that if this force is applied to the binary the softening parameter $\epsilon$ is set to 0 , and if this force is applied to the planet we exclude the material located inside the Roche lobe of the planet $R_{H}=a_{\mathrm{p}}\left(m_{\mathrm{p}} / 3 M_{\odot}\right)^{1 / 3}$. Previous simulations have demonstrated that $2 \mathrm{D}$ simulations of protoplanets embedded in disks give migration rates in good agreement with 3D results if $\epsilon=0.6 \mathrm{H}$ (Nelson \& Papaloizou 2004), which is why we adopt this value for the softening. As already mentioned, the last term $-\nabla \Phi_{\text {ind }}$ arises because the frame centrered on the binary centre of mass is not inertial. 


\section{Numerical setup}

\subsection{Numerical method}

The Navier-Stokes equations are solved using the hydrodynamic code GENESIS, which is basically a 2D ZEUS-like code. It uses a staggered mesh and solves the equations of motion for the disk by means of finite differences. The numerical method used in this code is spatially second-order and the implemented advection scheme is based on the monotonic transport algorithm (Van Leer 1977). Although the FARGO algorithm (Masset 2000) is implemented in GENESIS, it was not used for the simulations presented in this paper. The evolution of the planet and binary orbits are computed using a fifth-order Runge-Kutta integrator (Press et al. 1992).

GENESIS has already been employed in a project aimed at comparing several hydrocodes on the disk-planet interaction problem (de Val-Borro et al. 2006). In this context, the runs performed with the GENESIS code give similar results to those obtained using standard hydrodynamics codes such as ZEUS or NIRVANA.

For most of the calculations presented here, we use $N_{r}=$ 256 grid cells in radius and $N_{\phi}=380$ grid cells in azimuth. Some low resolution runs of binary-disk interactions using $N_{r}=128$ and $N_{\phi}=128$ grid cells have also been performed. These are long-term evolution calculations, aimed at showing that binarydisk interactions lead to an equilibrium state where the disk structure, as well as the binary eccentricity remain constant in time. This stationary state is obtained after about $5 \times 10^{5}$ binary orbits and would require several months of run-time to be reached at higher resolution.

\subsection{Computational units}

We adopt computational units in which the total mass of the binary is $M_{*}=1$, the gravitational constant is $G=1$, and the radius $r=2$ in the computational domain corresponds to $5 \mathrm{AU}$. The unit of time is $\Omega^{-1}=\sqrt{G M_{*} / a_{\mathrm{b}}^{3}}$, where $a_{\mathrm{b}}$ is the initial semi-major axis of the binary which is set to $a_{\mathrm{b}}=0.4$ in this work. This corresponds to an initial separation between the two stars of $\sim 1$ AU. If any planet is present in the disk, it is initially located at $r_{\mathrm{p}}=2.5$, which would correspond to $r_{\mathrm{p}}=6.2 \mathrm{AU}$ in physical units.

\subsection{Initial and boundary conditions}

In the model that we adopt for the simulations presented here, the disk aspect ratio is constant and equal to $H / r=0.05$, which is a typical value for protoplanetary disks. The inner edge of the computational domain is located at $r_{\text {in }}=0.5$ and the outer edge at $r_{\text {out }}=6$.

We use closed, reflecting boundary conditions at both the inner and outer radial boundaries. Because we are interested in long-term evolution runs, this ensures that the disk mass does not vary. There is no evidence of wave reflection at the inner boundary, in spite of the use of reflecting boundary conditions. This is because the inner boundary is located deep inside the tidally truncated cavity created by the binary, and as a consequence, the density there is very small. In order to avoid wave reflection/excitation at the outer boundary, we impose a low-density region from $r=4$ to $r=6$ using a taper function.

From $r=0.5$ to $r=4$, the initial surface density distribution is set to $\Sigma(r)=\Sigma_{0} r^{-1 / 2}$, where $\Sigma_{0}$ is defined in such a way that the disk would contain $0.01 M_{\odot}$ inside $10 \mathrm{AU}$ (assuming that the mass of the binary is $M_{*}=1 M_{\odot}$ ).

The anomalous viscosity in the disk, which probably arises from MHD turbulence in nature, is modelled using a standard "alpha" prescription for the effective kinematic viscosity $v=$ $\alpha c_{\mathrm{s}} H$ (Shakura \& Sunyaev 1973), where $c_{\mathrm{s}}$ is the isothermal sound speed. Canonical mass accretion rates of $\simeq 10^{-8} M_{\odot}$ per year observed to occur in T Tauri systems require $\alpha$ values in the range $10^{-2}-10^{-3}$. For the purposes of this study, however, we have adopted a lower value of $\alpha=10^{-4}$. The reason for this is that in earlier test simulations, larger values of $\alpha$ caused the binary semimajor axis to decrease too rapidly to allow a steady state disk structure and binary eccentricity to be established. Shrinkage of the binary orbit eventually causes the important 1:2 and 1:3 Lindblad resonances to be located interior to the inner boundary of the numerical grid rather than within its body, such that the simulations become unrealistic. The smaller value of $\alpha$ adopted prevents this from occuring and allows a steady state disk structure to be established (albeit after very long run times).

Due to the very long run times required to establish a quasisteady state for the binary-circumbinary disk system, we have only been able to consider a single binary mass ratio $q_{\mathrm{b}}=0.1$. The binary is initiated on a circular, Keplerian orbit with $a_{\mathrm{b}}=$ 0.4 in code units.

\section{Results}

\subsection{Evolution of the binary-disk system}

We begin by focusing on the interaction between the disk and the binary, and on the joint evolution of these two bodies. The aim of these simulations is to demonstrate that the system can reach a quasi-equilibrium state in terms of disk structure and orbital elements of the binary. This near-stationary state will be used as an initial condition for calculations dealing with the evolution of embedded planets in circumbinary disks.

\subsubsection{Theoretical expectations}

Before presenting the simulation results, we discuss the expected behaviour of the system based on previous numerical and theoretical work. Early work on close binary systems interacting with accretion disks focussed on the evolution of disks surrounding one of the binary components (the primary). Of particular interest was the superhump phenomenon observed in the so-called SU UMa subclass of Cataclysmic Variable systems. These systems have fairly extreme binary mass ratios $q_{\mathrm{b}} \simeq 0.1$, and the superhump phenomenon (characterised as a modulation of the lightcurve evolution) was interpreted as being due to a precessing, eccentric disk (e.g. Whitehurst 1988; Hirose \& Osaki 1990; Lubow 1991). Lubow (1991) presented a fluid dynamical theory that explained the origin of the eccentric disk as being due to an instability at the 3:1 Lindblad resonance generated by non linear mode coupling, leading to the growth of disk eccentricity for a circular binary orbit.

The interaction between massive companions (giant planets and brown dwarfs) and accretion disks in which they were initially embedded was considered by Papaloizou et al. (2001). Here the tidal interaction creates an inner cavity within which the primary star and companion orbit, and as such these systems are similar to the binary plus circumbinary disks that we are concerned with in this paper. Papaloizou et al. (2001) showed that a massive companion on a circular orbit could cause the surrounding disk to become eccentric. The origin of the eccentricity was 
found to be due to a similar instability mechanism to that proposed by Lubow (1991), namely non linear coupling between an initial $m=1$ eccentric disturbance in the disk and the $m=1$ component of the binary potential leading to an $m=2$ wave being excited at the 1:3 resonance in the circumbinary disk. Based on this we expect to see the circumbinary disk in our simulations become eccentric. Eccentricity growth will saturate when viscous damping matches the eccentricity forcing rate. Secular interaction between the eccentric disk and binary should force the binary to become eccentric also.

There are important resonant interactions between the disk and binary that can lead to modification of the binary orbital elements. For a binary on a modestly eccentric orbit interaction at outer Lindblad resonances is expected to cause a decay of the semimajor axis in a viscous disk, where the decay rate depends on the disk viscosity. Interaction at eccentric Lindblad resonances is expected to cause growth of the eccentricity. Working to first order in the binary eccentricity, $e_{\mathrm{b}}$, the 1:2 corotation resonance should induce eccentricity damping and the $1: 3$ eccentric Lindblad resonance should cause eccentricity growth. If the disk is tidally truncated beyond the 1:2 Lindblad resonance, as is expected for massive companions (e.g. Artymowicz 1992; Lin \& Papaloizou 1993), then eccentricity growth is expected. Thus we expect the eccentricity of the binary system to grow in our simulations, provided the disk inner cavity does not extend beyond the $1: 3$ resonance. Should this occur the eccentricity should still grow, but at a lower rate since higher order resonances will be required to drive the eccentricity. At the present time there is not a well developed theory that can be used to predict when the binary eccentricity should saturate. We note, however, that as the eccentricity grows the interaction at the most significant eccentric Lindblad resonances may become increasingly non linear such that they saturate (i.e. the density there is decreased), causing the growth rate to slow.

We see from the above discussion that we expect there to be both secular and resonant interactions occuring between the disk and binary, leading to the growth of their eccentricity. When the angular momentum content of disk and binary are similar then we expect that they may participate in a joint secular mode in which they precess at the same rate (Papaloizou 2002). In fact the angular momentum in the binary exceeds that in the disk by about a factor of 4 in our simulations, so the existence of a joint mode is probably marginal. When their apsidal lines are misaligned the disk and binary will exert secular torques leading to changes in their eccentricities (by analogy with the JupiterSaturn system). If the apsidal lines become closely aligned, however, then these torques will diminish. Thus, we expect that a steady state configuration will consist of an eccentric binary system surrounded by an eccentric disk precessing at the same rate in a prograde direction with with apsidal lines closely aligned.

\subsubsection{Simulation results}

We now discuss the simulation results for the binary plus circumbinary disk runs. The upper panel of Fig. 1 shows a snapshot of the disk surface density after $1.2 \times 10^{5}$ binary orbits. Here, the disk surrounds a binary with mass ratio $q_{\mathrm{b}}=0.1$. The binary initially evolves on a circular orbit and the initial separation between the two stars is $a_{\mathrm{b}}=0.4$. The lower panel displays the corresponding azimuthal average of the disk surface density, as well as the initial surface density profile. Torques exerted by the binary truncate the inner edge of the disk. We find that the gap size is $\sim 2.5 a$, which is consistent with analytical estimates from Artymowicz \& Lubow (1994).

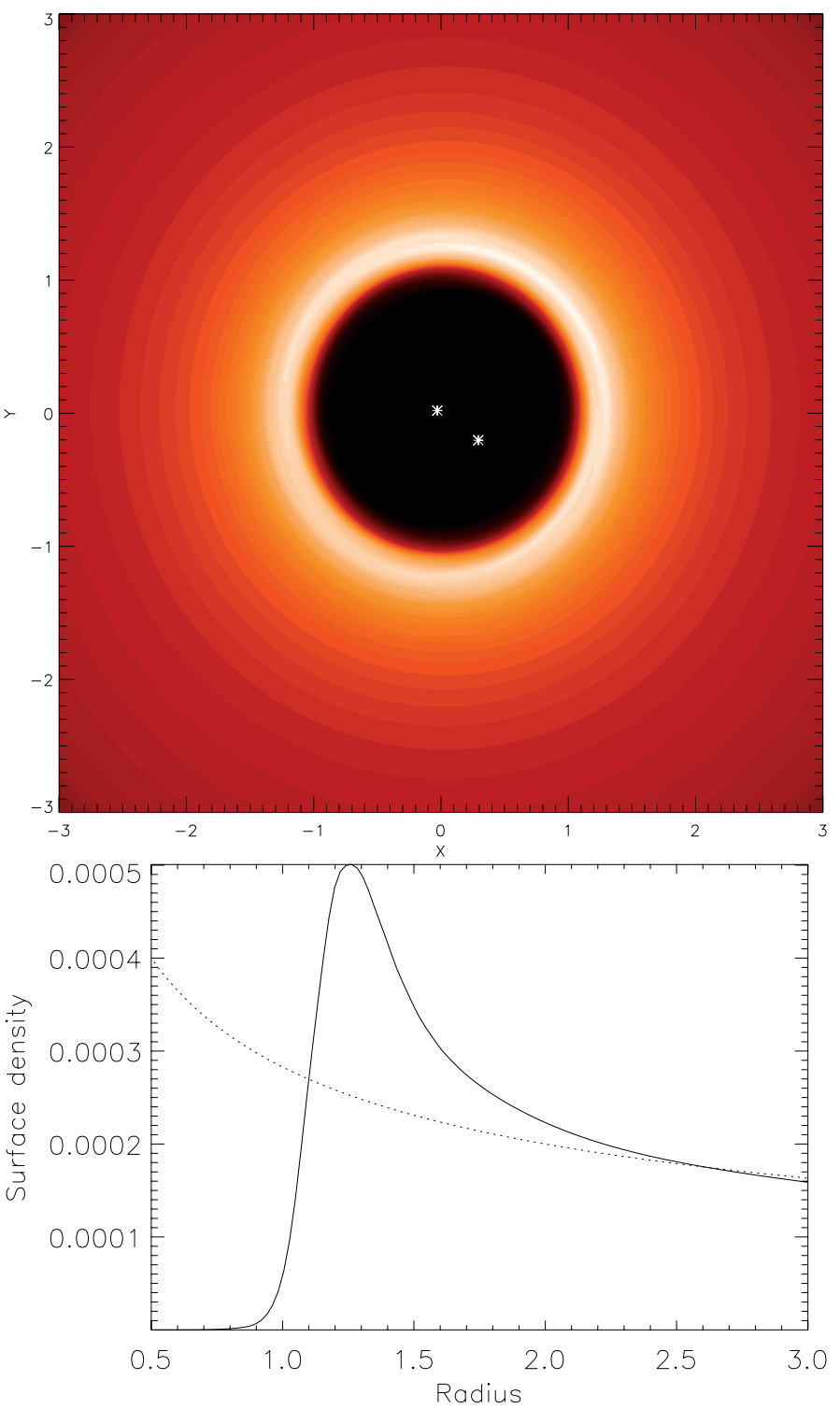

Fig. 1. The upper panel shows a snapshot of disk surface density after $1.2 \times 10^{5}$ binary orbits. The lower panel displays the azimuthal average of the surface density at the same time.

Figure 2 shows the evolution of the binary semi-major axis and eccentricity as a function of time, deduced from lowresolution simulations with $128 \times 128$ grid cells. As a result of angular momentum being transferred to the disk, the binary separation shrinks at a rate $\mathrm{d} a / \mathrm{d} t \sim 10^{-8}$. This value is consistent with the orbital decay that we would expect from analytical estimates (i.e. Armitage \& Natarajan 2005):

$\frac{\mathrm{d} a}{\mathrm{~d} t} \propto-\alpha\left(\frac{h}{r}\right)^{2} \frac{M_{\mathrm{d}}}{M_{2}} \Omega a$

where $M_{\mathrm{d}}$ is the disk mass and $M_{2}$ the mass of the secondary. The orbital decay of the binary is slow enough that the 1:3 commensurability at $r \sim 2.08 a_{\mathrm{b}}$, which corresponds to the eccentric Lindblad resonance which is expected to be important in determining the evolution of the binary orbit, always resides inside the computational domain. This makes it possible to do long-term evolution runs over $\sim 10^{5}$ binary orbits, at least at lowresolution. We note that the location of the 1:3 resonance is just 

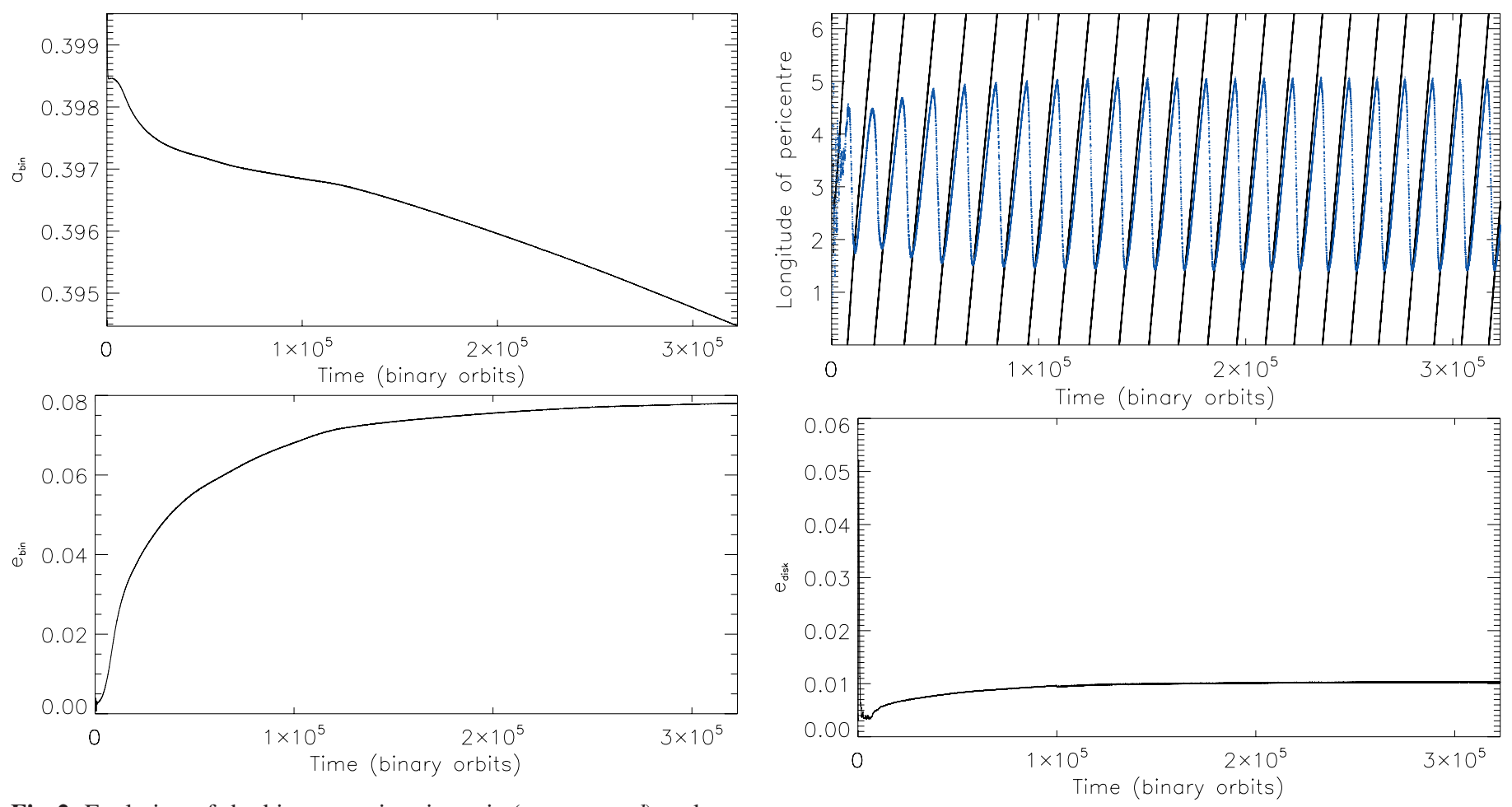

Fig. 2. Evolution of the binary semi-major-axis (upper panel) and eccentricity (lower panel) as a function of time, for low-resolution simulations with $128 \times 128$ grid cells.

at the base of the gap in Fig. 1, showing that the density there is quite low.

The lower panel in Fig. 2 shows that the binary eccentricity, after some transients lasting for $\sim 5 \times 10^{3}$, grows until it saturates at $e \sim 0.08$. The initial eccentricity growth is due to the resonant nature of the interaction between the disk and the binary. This interaction is expected to occur mainly at the location of the 1:3 outer Lindblad resonance, which promotes eccentricity growth. As time goes by and the eccentricity grows, there is evidence that this resonance saturates due to non linear effects (i.e. the density at the resonance is decreased), and this saturation combined with higher-order resonances coming into play (Artymowicz 1992) causes the eccentricity to reach a steady value. We also need to consider the secular interaction between disk and binary, as this also plays an important role.

We plot the evolution of the longitudes of pericentre for the disk $\left(\omega_{\mathrm{d}}\right)$ and binary $\left(\omega_{\mathrm{b}}\right)$ in the upper panel of Fig. 3. The disk eccentricity evolution is plotted in the lower panel. The densityweighted $\omega_{\mathrm{d}}$ is calculated according to the definition:

$\omega_{\mathrm{d}}=\frac{\int_{0}^{2 \pi} \int_{r_{\mathrm{in}}}^{r_{\max }} \Sigma \omega_{\mathrm{c}} \mathrm{d} S}{\int_{0}^{2 \pi} \int_{r_{\mathrm{in}}}^{r_{\max }} \Sigma \mathrm{d} S}$

where $\omega_{\mathrm{c}}$ is the longitude of pericentre computed inside each grid cell, and $r_{\max }=3$ for the simulations presented here. Test calculations have shown that $\omega_{\mathrm{d}}$ does not depend strongly on the value of $r_{\text {max }}$. The figure shows that both the binary and disk precess in a prograde sense, and that at late times the apsidal lines of the disk and binary come into almost perfect alignment. This alignment coincides with the time when the binary eccentricity reaches a steady value, showing that the secular interaction between disk and binary has a significant effect on the eccentricity evolution of the binary.

Fig. 3. The upper panel shows, as a function time, the evolution of the longitudes of pericentre for both the binary (black line) and the disk (blue line), deduced from a low-resolution simulation with $128 \times$ 128 grid cells. The lower panel displays the evolution of the disk eccentricity.

The disk eccentricity, $e_{\mathrm{d}}$, shown in the lower panel of Fig. 3, is defined in the simulations by:

$e_{\mathrm{d}}=\frac{\int_{0}^{2 \pi} \int_{r_{\mathrm{in}}}^{r_{\max }} \Sigma e_{\mathrm{c}} \mathrm{d} S}{\int_{0}^{2 \pi} \int_{r_{\mathrm{in}}}^{r_{\max }} \Sigma \mathrm{d} S}$

where $e_{\mathrm{c}}$ is the eccentricity computed at the center of each grid cell. The disk eccentricity evolves similarly to the binary eccentricity and saturates at the relatively small value of $e_{\mathrm{d}} \sim 0.01$.

In line with the expectations outlined in Sect. 4.1.1, the disk and binary become eccentric. They achieve a steady state configuration when the apsidal lines of disk and binary are aligned and both disk and binary precess at the same rate in a prograde sense.

Figures 4 and 5 show the results of higher resolution simulations with $256 \times 380$ grid cells. Figure 4 shows the evolution of the binary semimajor axis and eccentricity, and Fig. 5 shows the longitudes of pericentre of disk and binary (upper panel), and the disk eccentricity (lower panel). Because of their expensive computing time, high-resolution simulations cannot be run for the same length of time as low-resolution ones, and we have been unable to run this simulation until the binary and disk eccentricities have completely saturated. Nevertheless, we can see by comparing the results from the low and high resolutuion runs, that the two simulations are in excellent agreement. At a time of $\simeq 1.5 \times 10^{5}$ binary orbits, we see that binary eccentricity is $e_{\mathrm{b}} \simeq 0.07$ in both cases, and the disk eccentricity is $\simeq 0.01$ in each case. We see from Fig. 5 that the apsidal lines of the disk and the binary are almost aligned at the end of the simulations, such that it appears that the system is close to an equilibrium state. It is therefore reasonable to use the final result of this 

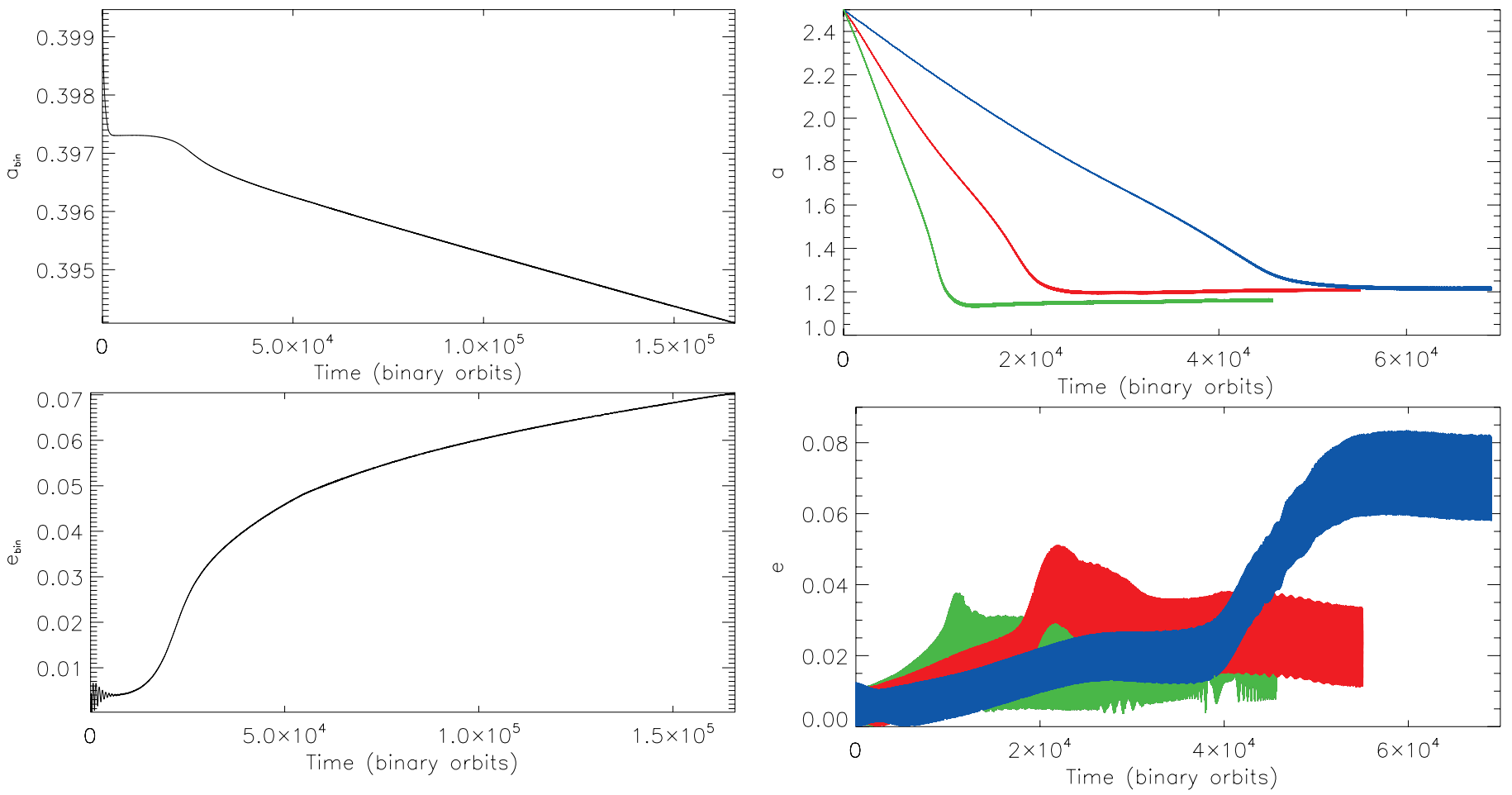

Fig. 4. Same as Fig. 2 but for high-resolution simulations with $256 \times 380$ grid cells.
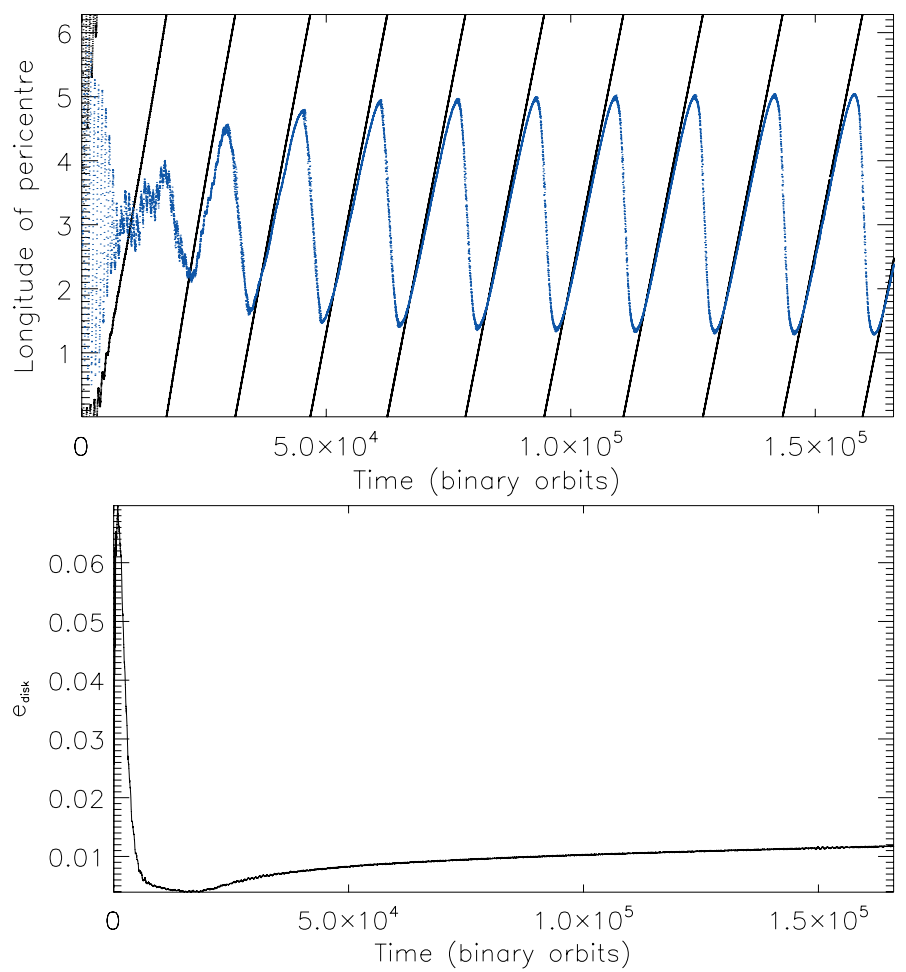

Fig. 5. Same as Fig. 3 but for high-resolution simulation with $256 \times 380$ grid cells.

high-resolution run as the initial condition for simulations dealing with the evolution of protoplanets embedded in circumbinary disks. We present the results of such simulations in the following section.

Fig. 6. The upper panel of this figure shows the semi-major axis of the planets as a function of time, for three different planet masses. The lower panel displays the eccentricity evolution of all three planets.

\subsection{Migration of planets in circumbinary disks}

We have performed three simulations with embedded protoplanets of mass $m_{\mathrm{p}}=5,10$ and $20 M_{\oplus}$, respectively. These masses are small enough that we expect the interaction between the disk and the planet to be linear, although the $m_{\mathrm{p}}=20 M_{\oplus}$ case is marginal. We insert these planets in the high resolution circumbinary disk on circular orbit at $r=2.5$, and let them evolve under the influence of both the disk and binary. The results are presented in Fig. 6, which shows the evolution of the planetary semi-major axis $a_{\mathrm{p}}$ (top panel) and eccentricity $e_{\mathrm{p}}$ (bottom panel).

As expected, each protoplanet initially undergoes a period of inward migration due to the torques exerted by the disk. We find migration rates consistent with analytical estimates for type I migration (e.g. Tanaka et al. 2002). At later times, all the protoplanets stop their migration at about the same distance from the binary, located at $r \sim 1.1-1.2$. We discuss the reasons for this stalled migration below. As the planet migrates in, $e_{\mathrm{p}}$ slowly increases because the damping of $e_{\mathrm{p}}$ is not strong enough to counterbalance the eccentricity growth due to the interaction with binary. We see that the final eccentricity is inversely proportional to the protoplanet mass, simply because the disk induced eccentricity damping rate is also inversely proportional to the planet mass (e.g. Papaloizou \& Larwood 2000; Tanaka \& Ward 2004).

Figure 7 shows the evolution of the longitudes of pericentre of the binary, disk and protoplanet for the run with $m_{\mathrm{p}}=5 M_{\oplus}$. We see that the disk and binary are close to alignment, and precess in a prograde direction at more of less the same rate. The protoplanet eventually precesses at the same rate, but maintains a constant angle of misaligment equal to about 0.5 radians.

Figure 8 shows a snapshot of the surface density and the corresponding azimuthal average after $2 \times 10^{4}$ binary orbits for the simulation with $m_{\mathrm{p}}=20 M_{\oplus}$, when the planet has stopped its migration. 


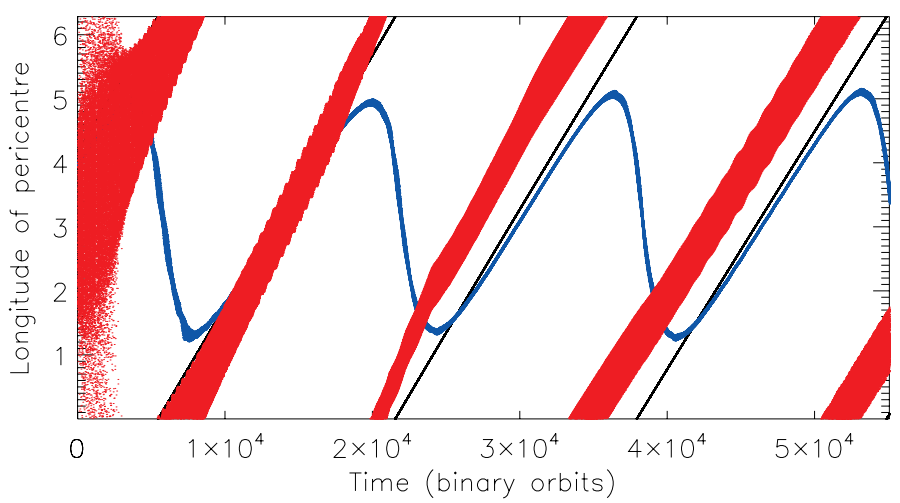

Fig. 7. This shows the evolution of the longitudes of pericentre for the binary (black line), disk (blue line) and planet (red line).

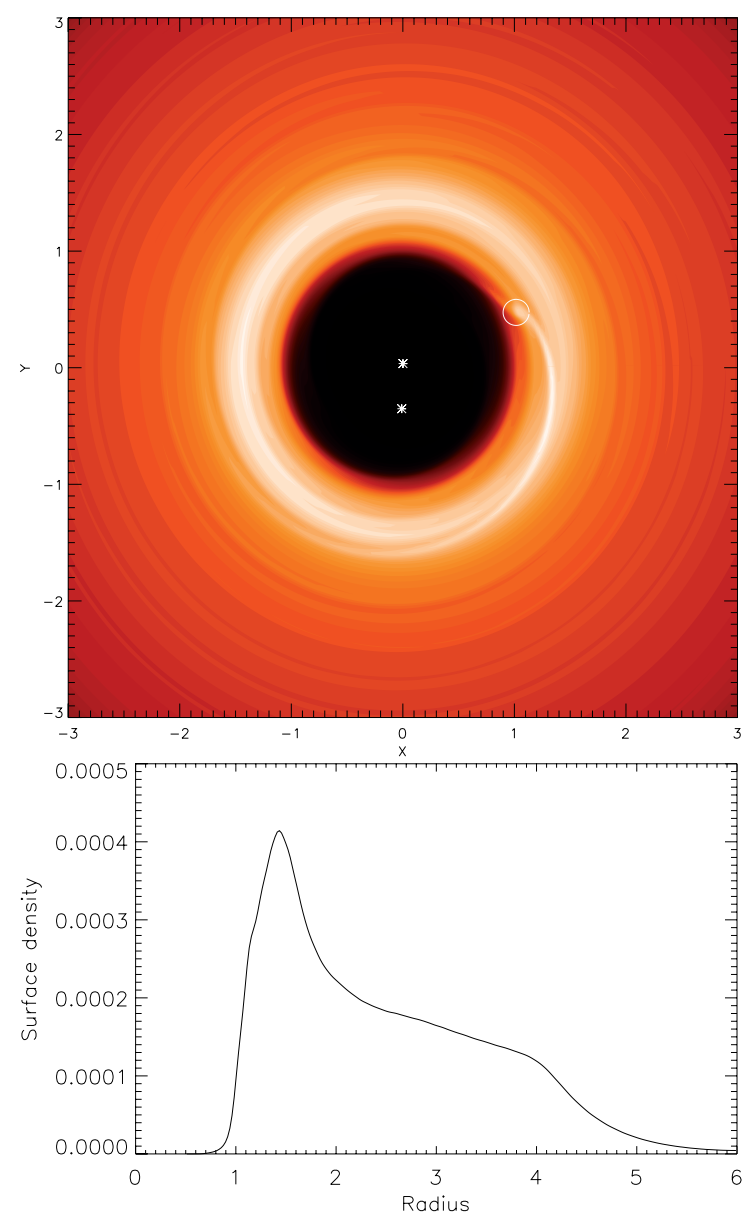

Fig. 8. The upper panel of this figure shows a snapshot of disk surface density after $2 \times 10^{4}$ binary orbits. At this time, the migration of the planet is stopped. The lower panel shows the azimuthal average of the disk surface density at the same time.

In order to explain the stalling of migration, we have searched for mean motion resonances between the protoplanets and binary system. Indeed the semimajor axis $a_{\mathrm{p}} \simeq 1.2$ at which the planets stall is very close to the 5:1 mean motion resonance with the binary. We found no evidence, however, that the protoplanets are in mean motion resonance. Moreover, we find that migration stops even in test simulations in which the planet does not feel the force due to the secondary star. This is strong evidence that the effect we observe is not due to the binary but instead arises because of the disk.
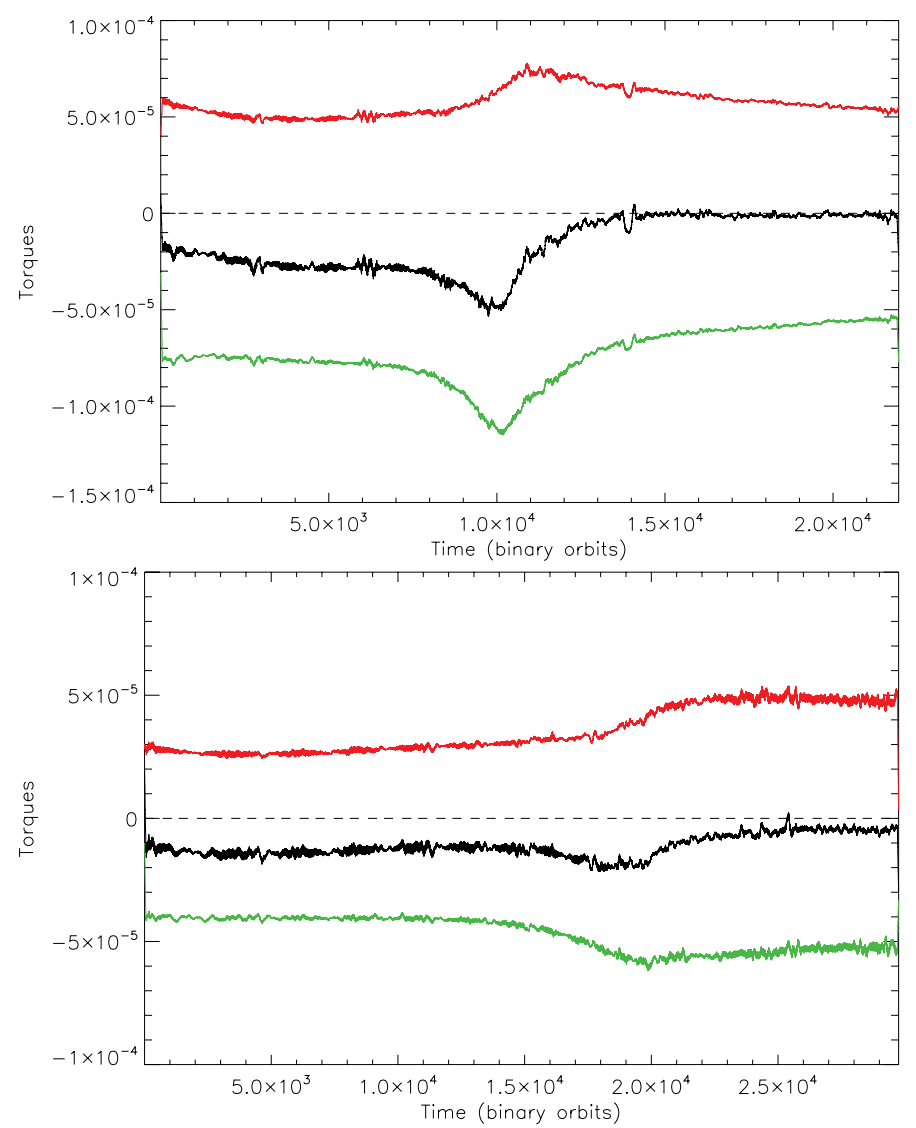

Fig. 9. This figure shows respectively the torques exerted by the disk on a $20 M_{\oplus}$ (upper panel) and a $10 M_{\oplus}$ (lower panel) as a function of time. The green (resp. red) line corresponds to the torques exerted by the inner (resp. outer) disk. The black line corresponds to the total torque.

Figure 9 shows the total torques exerted by the disk on the planet as well as the torques due to the disk interior to $a_{\mathrm{p}}$ and exterior to $a_{\mathrm{p}}$ for the simulations with $m_{\mathrm{p}}=20$ and $10 M_{\oplus}$. As expected, the outer torques are initially larger than the inner ones, corresponding to the inward migration of the planet. The $10 M_{\oplus}$ planet undergoes a period of accelerated migration at around $t=2 \times 10^{4}$ binary orbits while the $20 M_{\oplus}$ planet undergoes accelerated migration at around $t=10^{4}$ binary orbits. This arises when the planets approach the tidally maintained inner cavity where the disk surface density is higher, resulting in an increased differential Lindblad torque acting on the planet. Because of the buffer action of the pressure gradient (Ward 1997), the strong density gradient at $r \sim 1.5$ (see lower panel of Fig. 8) acts in the same way. Furthermore, it leads to a strong negative corotation torque, which promotes also an accelerated migration. After $\sim 2 \times 10^{4}$ binary orbits, the total torque exerted on the planet goes to zero, which is consistent with the stopping of migration. The outer torques couterbalance the inner ones exactly from this time onward.

The cancellation of the total torque appears to arise because the corotation torques increase in regions of strong positive surface density gradients, and can become strong enough to cancel out the differential Lindblad torque. This occurs when the planet enters the inner cavity maintained by the binary tidal torques. This effect was originally discovered by Masset et al. (2006), who have shown that in a disk with a surface density transition of about $50 \%$ occuring on a radial length scale equivalent to 35 disk vertical scale heights, corotation torques (which are positive) can equal or exceed the differential Lindblad torque (which 


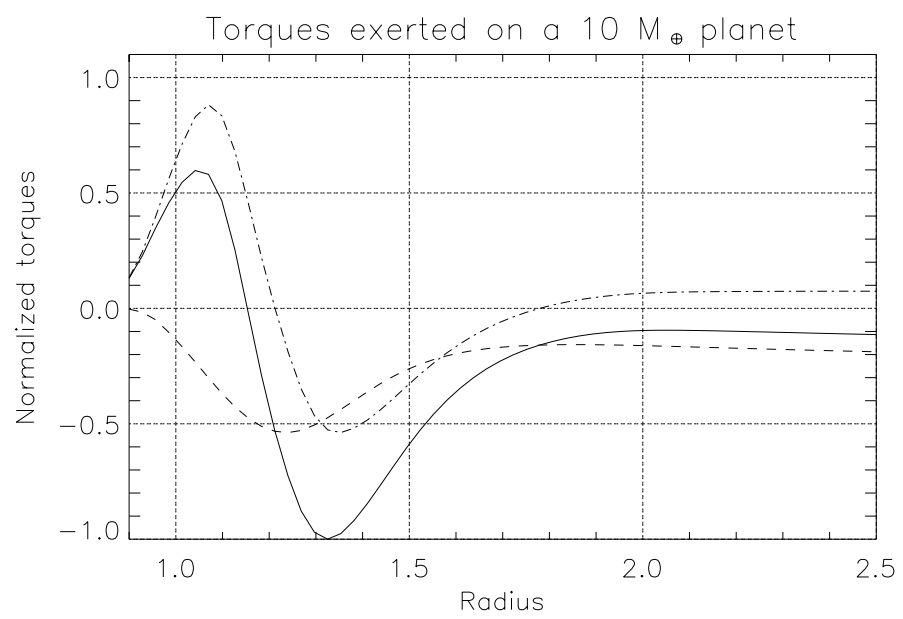

Fig. 10. This figure shows the semi-analytic torques exerted on a $10 M_{\oplus}$ protoplanet as a function of the orbital radius. The dashed line represents the Lindblad torques and the dot-dashed line represents the corotation torques. The solid line shows the total (Lindblad + corotation) torques.

is negative) near the transition. They found that a protoplanet undergoing type I migration can reach a fixed point at the transition where the total torque cancels out and where planetary migration stops. This effect comes into play provided that the length scale of the surface density jump $\lambda$ is $\leq 8 H C / 3$, where $H$ is the disk scale height at the transition and $C$ is a constant of order unity which depends on the profile of the density jump. In the simulations presented in this work, $\lambda \sim 2 H$, which indicates that it is indeed the corotation torque which is responsible for the observed stalling of migration near the gap edge.

In order to clearly demonstrate that this effect is at work here, we have computed semi-analytically the corotation torques as well as the Lindblad torques exerted on the planet. Corotation and Lindblad torques can be computed in the linear regime from standard formulae (e.g. Goldreich \& Tremaine 1980) using the surface density and rotation profile obtained in our simulations. Results of these calculations for a $10 M_{\oplus}$ planet are displayed in Fig. 10 which clearly shows that the corotation torques can be larger that the Lindblad torques near the gap edge. In agreement with Masset et al. (2006), we find also that the total torque exhibits a fixed point at $r \sim 1.15$ where the corotation and Lindblad torques cancel each other. This value corresponds approximately to the location where the planet stops its migration in the hydrodynamical simulations. For $m_{\mathrm{p}}=20 M_{\oplus}$, the location where the planet stops exhibits a small deviation with respect to the other cases. This is probably due to the onset of non-linear effects which significantly alter the surface density profile and the corotation torque.

\subsection{Corotation torque saturation and turbulence}

The trapping of planets at the edge of the tidally truncated cavity in circumbinary discs requires the action of corotation torques, and it is well known that these can saturate in the absence of viscosity or some other dissipative mechanism that is able to maintain the surface density gradient in the vicinity of the planet (e.g. Ogilvie \& Lubow 2003; Masset et al. 2006). It is worth noting that in a circumbinary disc the edge of the cavity is maintained through a balance between tidal torques and viscous stresses, such that even in a disc with very low viscosity it is possible that saturation of the corotation torques may be prevented by the tidal stresses due to the binary acting in the disc, rather than through the viscosity itself.

In the simulations we present in this paper the viscous stress parameter $\alpha=10^{-4}$, and this value is probably large enough to prevent corotation torque saturation since it is consistent with the minimum value required to prevent saturation derived in Masset et al. (2006). The origin of anomalous viscosity in accretion discs is thought to be MHD turbulence generated via the magnetorotational instability (MRI) (Balbus \& Hawley 1991). Explicitly simulating a circumbinary disc that is undergoing MHD turbulence is beyond current computational capabilities, but we note that ideal MHD simulations of the MRI for zero net flux magnetic fields typically yield values of $\alpha$ in the range $10^{-3}$ $10^{-2}$ (Brandeburg et al. 2005; Hawley et al. 2006; Papaloizou $\&$ Nelson 2003). These values that are clearly large enough to prevent saturation of corotation torques. Questions remain about the level of MHD turbulence that can be sustained in protostellar discs, since the ionisation fraction is expected to be very low in significant regions of these discs (Gammie 1996). The primary source of ionisation is likely to be X-rays from the central protostar(s) (Glassgold et al. 1997), and chemical models of discs irradiated by X-rays indicate that regions close to the star and the upper disc layers will be sufficiently ionised to maintain MHD turbulence (Fromang et al. 2002; Ilgner \& Nelson 2006). In the case of a circumbinary case with tidally truncated cavity, the cavity edge is face-on to the binary and will therefore be exposed to a significant X-ray flux, so we should expect that this region of the disc will be able to sustain MHD turbulence that could in principle prevent the saturation of corotation torques.

It is worth noting that there are outstanding issues about how corotation torques actually operate in a turbulent disc. First, it has been shown that low mass planets in turbulent discs experience stochastic forcing due to turbulent density fluctuations (e.g. Nelson \& Papaloizou 2004; Nelson 2005), and these may be strong enough to cause the planet to "random walk" across the edge of the tidally truncated cavity where the corotation torques operate to prevent inward planet migration. In addition, the operation of corotation torques for a $20 M_{\oplus}$ protoplanet requires the fluid streamlines to form horseshoe-like orbits in the corotation region, and it remains unclear whether these horseshoe streamlines actually exist in a turbulent protoplanetary disc. These issues are of relevance to the effectiveness of corotation torques that operate in discs around single stars as well as in circumbinary discs, and will be explored in future publications.

\section{Summary and conclusion}

In this paper, we have presented hydrodynamical simulations of low-mass planets embedded in a circumbinary disk. The planets have masses ranging from 5 to $20 M_{\oplus}$ and evolve under the forces exerted by the disk and binary.

The central binary has mass ratio $q_{\mathrm{b}}=0.1$. Values for the binary eccentricity $e_{\mathrm{b}}$ and semi-major axis $a_{\mathrm{b}}$ are determined self-consistently from simulations of an initially circular binary with $a_{\mathrm{b}}=0.4$ interacting with a circumbinary disk. In agreement with expectations, a cavity is formed at the inner edge of the disk because of the tidal torques due to the binary. $a_{\mathrm{b}}$ continually decreases while $e_{\mathrm{b}}$ increases with time. We find that the final outcome of the interaction between the disk and the binary is a configuration where the disk and binary eccentricity reach a quasi-steady state. This occurs when the disk and binary precess in a prograde sense at the same rate with the apsidal lines of the disk and binary being aligned. In this quasi-steady state, 
$e_{\mathrm{b}} \sim 0.08$ and the inner edge of the disc is truncated at $\sim 2.5 a_{\mathrm{b}}$, where $a_{\mathrm{b}} \sim 0.4$.

The protoplanets we considered are inserted in the disk once this equilibrium configuration has been approched. After a period of type I inward migration, all the protoplanets were found to stop their migration at about the same location $r_{\mathrm{p}} \sim 1.15-1.2$, which is close to the edge of the inner cavity. Apparently, this behaviour arises because positive corotation torques cancel the negative Lindblad torques at this point in the disk, in agreement with the simulations of Masset et al. (2006) who considered the migration of planets in disks with surface density transitions. We examined the total torques exerted by the disk on the planet which were found to cancel as soon as the migration stalls. In addition, we computed semi-analytical values for the corotation and Lindblad torques. This analysis shows that, depending on the planet mass, migration should stop at $r_{\mathrm{p}} \sim 1.2$, which is in good agreement with the results of the simulations.

These results have a number of interesting consequences for planet formation in circumbinary disks. According to Holman \& Wiegert (1999), the critical semi-major axis for dynamical stability of a planet orbiting a binary with $e_{\mathrm{b}} \sim 0.08$ and $q_{\mathrm{b}}=0.1$ is $a_{\mathrm{p}} \sim 2.3 a_{\mathrm{b}}$. The planets in our simulations stall at a distance of $a_{\mathrm{p}} \sim 3 a_{\mathrm{b}}$. This suggests that migration stops at a location where the planet should be stable long after the dispersal of the circumbinary disk. From an observational point of view, it means that the location corresponding to the cavity edge of the precursor circumbinary disk appears to be an excellent place to look for low mass planets in close binary systems.

The final outcome of the system remains unclear, however, if the protoplanet grows to become a giant planet. As the protoplanet grows, gap formation can exclude the material from the coorbital region, cancelling the effects of the corotation torques. Once the gap is cleared, the planet should migrate inward again on a time scale consistent with the type II regime (e.g. Lin \& Papaloizou 1993; Nelson et al. 2000). Trapping into the 4:1 resonance with the binary seems to be the most likely fate of a giant planet interacting with a circumbinary disk (Nelson 2003). The evolution of protoplanets which can accrete gas from the disk will be the subject of a future publication.

A number of other issues remain to be resolved, which will be the subject of future papers. The first is how multiple low mass protoplanets will interact if they form at large distance from the binary and successively migrate toward the cavity edge. It remains an open question whether or not they will merge in this region to form a larger body, become locked in mean motion resonances, or simply undergo dynamical scattering. Also, the ability of planetesimals to accrete in circumbinary disks needs to be examined, with particular care being taken to simulate the structure of the circumbinary disk. It seems unlikely that planets will form near the cavity edge, but presumably there is a location outside of this which remains benign to planetary formation.
Once formed, these planets will then migrate inward toward the binary in the manner described in this paper.

Acknowledgements. The simulations performed in this paper were performed on the QMUL High Performance Computing facility purchased under the SRIF iniative, and on the UK Astrophysical Fluids Facility.

\section{References}

Armitage, P. J., \& Natarajan, P. 2005, ApJ, 634, 921

Artymowicz, P. 1992, PASP, 104, 769

Artymowicz, P., \& Lubow, S. H. 1994, ApJ, 421, 651

Balbus, S. A., \& Hawley, J. F. 1991, ApJ, 376, 214

Boss, A. P. 2006, ApJ, 641, 1148

Brandenburg, A., Nordlund, A., Stein, R. F., \& Torkelsson, U. 1995, ApJ, 446, 741

Chambers, J. E. 2006, ApJ, 652, L133

Cuzzi, J. N., Hogan, R. C., Paque, J. M., \& Dobrovolskis, A. R. 2001, ApJ, 546, 496

de Val-Borro, M., Edgar, R. G., Artymowicz, P., et al. 2006, MNRAS, 370, 529

Duquennoy, A., \& Mayor, M. 1991, A\&A, 248, 485

Dutrey, A., Guilloteau, S., \& Simon, M. 1994, A\&A, 286, 149

Eggenberger, A., Udry, S., \& Mayor, M. 2004, A\&A, 417, 353

Fromang, S., Terquem, C., \& Balbus, S. A. 2002, MNRAS, 329, 18

Gammie, C. F. 1996, ApJ, 457, 355

Glassgold, A. E., Najita, J., \& Igea, J. 1997, ApJ, 480, 344

Goldreich, P., \& Tremaine, S. 1980, ApJ, 241, 425

Günther, R., \& Kley, W. 2002, A\&A, 387, 550

Günther, R., Schäfer, C., \& Kley, W. 2004, A\&A, 423, 559

Haisch, K. E., Jr., Lada, E. A., \& Lada, C. J. 2001, ApJ, 553, L153

Hawley, J. F., Gammie, C. F., \& Balbus, S. A. 1996, ApJ, 464, 690

Hirose, M., \& Osaki, Y. 1990, PASJ, 42, 135

Holman, M. J., \& Wiegert, P. A. 1999, AJ, 117, 621

Ilgner, M., \& Nelson, R. P. 2006, A\&A, 445, 205

Lin, D. N. C., \& Papaloizou, J. C. B. 1993, Protostars and Planets III, 749

Lubow, S. H. 1991, ApJ, 381, 259

Masset, F. 2000, A\&AS, 141, 165

Masset, F. S., Morbidelli, A., Crida, A., \& Ferreira, J. 2006, ApJ, 642, 478

Moriwaki, K., \& Nakagawa, Y. 2004, ApJ, 609, 1065

Mugrauer, M., \& Neuhäuser, R. 2005, MNRAS, 361, L15

Mugrauer, M., Neuhaeuser, R., \& Mazeh, T. 2007, A\&A, 469, 755

Nelson, R. P. 2003, MNRAS, 345, 233

Nelson, R. P. 2005, A\&A, 443, 1067

Nelson, R. P., \& Papaloizou, J. C. B. 2004, MNRAS, 350, 849

Nelson, R. P., Papaloizou, J. C. B., Masset, F., \& Kley, W. 2000, MNRAS, 318, 18

Ogilvie, G. I., \& Lubow, S. H. 2003, ApJ, 587, 398

Papaloizou, J. C. B. 2002, A\&A, 388, 615

Papaloizou, J. C. B., \& Larwood, J. D. 2000, MNRAS, 315, 823

Papaloizou, J. C. B., \& Nelson, R. P. 2003, MNRAS, 339, 983

Press, W. H., Teukolsky, S. A., Vetterling, W. T., \& Flannery, B. P. 1992, Numerical recipes in FORTRAN. The art of scientific computing (Cambridge: University Press), 2nd Ed.

Quintana, E. V., \& Lissauer, J. J. 2006, Icarus, 185, 1

Shakura, N. I., \& Sunyaev, R. A. 1973, A\&A, 24, 337

Tanaka, H., \& Ward, W. R. 2004, ApJ, 602, 388

Tanaka, H., Takeuchi, T., \& Ward, W. R. 2002, ApJ, 565, 1257

Udry, S., Mayor, M., Naef, D., et al. 2002, A\&A, 390, 267

Van Leer, B. 1977, J. Comp. Phys., 23, 276

Ward, W. R. 1997, Icarus, 126, 261

Whitehurst, R. 1988, MNRAS, 232, 35

Zucker, S., \& Mazeh, T. 2002, ApJ, 568, L113 